\title{
On the Concept of Representations of Prime Numbers and Prime Products
}

\author{
Peter Bissonnet
}

\author{
Received: December 1, 2017 Accepted: December 21, 2017 Online Published: January 29, 2018 \\ doi:10.5539/jmr.v10n1p132 \\ URL: https://doi.org/10.5539/jmr.v10n1p132
}

\begin{abstract}
The author used to think that the only representation of prime numbers, etc. was the prime number double helix representation. However, in 2011, the author published a paper which puzzled him and it eventually dawned upon him that there was more than one representation of prime numbers. The second representation will be referred to as the hyperbolic representation. The third representation will be called the parabolic representation and is related to the hyperbolic representation. The fourth representation is the triangular representation and is related to the hyperbolic representation. Both of the primary representations, the double helix and the hyperbolic both reject that 2 and 3 are prime numbers. The hyperbolic representation is shown to be related to Lorentz-like transformations.
\end{abstract}

Keywords: double helix, prime numbers, prime products, 2 and 3 not prime numbers. Lorentz transformation

\section{Introduction}

The prime numbers and prime products seem to be somewhat of a changeling or perhaps more like the elephant described by the proverbial blind men, each giving an incomplete description by examining the tail, the feet, the trunk, and the ear. The author was puzzled that so many concepts of prime numbers and prime products exist around the world. Whether they are random or form a pattern, do they obey a spiral, wave (Hahn, H. K., 2008), straight line, etc. In order to account for these varied ideas, the author thinks that prime numbers and prime products can be found in many different so-called representations. The author thinks that he has found four such representations.

\section{The Double Helix Representation (Bissonnet, P., 2006)}

The reader is asked to imagine an infinite row, seven column array. Further, imagine that all of the non-prime numbers have been blanked out. One ends up with a set of double lines of prime numbers extending from column 1 to column 6 , except for row 1 in which the number 7 is in column 7 (see Fig. 1). The blanked-out cells along the double lines are either simple prime products or multiple prime products. $6 \mathrm{~s}-1\left(\mathrm{H}_{1}\right.$ or helix 1$)$ describes the numbers along the top line, while $6 \mathrm{~s}$ $+1\left(\mathrm{H}_{2}\right.$ or helix 2 ) describes the numbers along the second line of each pair of lines, with s an integer and $s \geq 1$ (see Table 1). The lines can extend up into column 7, but only the simple and multiple prime products involving 7 will appear. The multiplication rule that is obeyed is the following:

$$
(6 m+a)(6 n+b)=6 r+c
$$

where $\mathrm{m}, \mathrm{n}$ and $\mathrm{r}$ are integers $\geq 1$, and $\mathrm{a}, \mathrm{b}$, and $\mathrm{c}$ are +1 or -1 . Another rule of the multiplication is that $\mathrm{H}_{1} \otimes \mathrm{H}_{1}=\mathrm{H}_{2} \otimes$ $\mathrm{H}_{2}=\mathrm{H}_{2}$ and $\mathrm{H}_{1} \otimes \mathrm{H}_{2}=\mathrm{H}_{2} \otimes \mathrm{H}_{1}=\mathrm{H}_{1}$. 


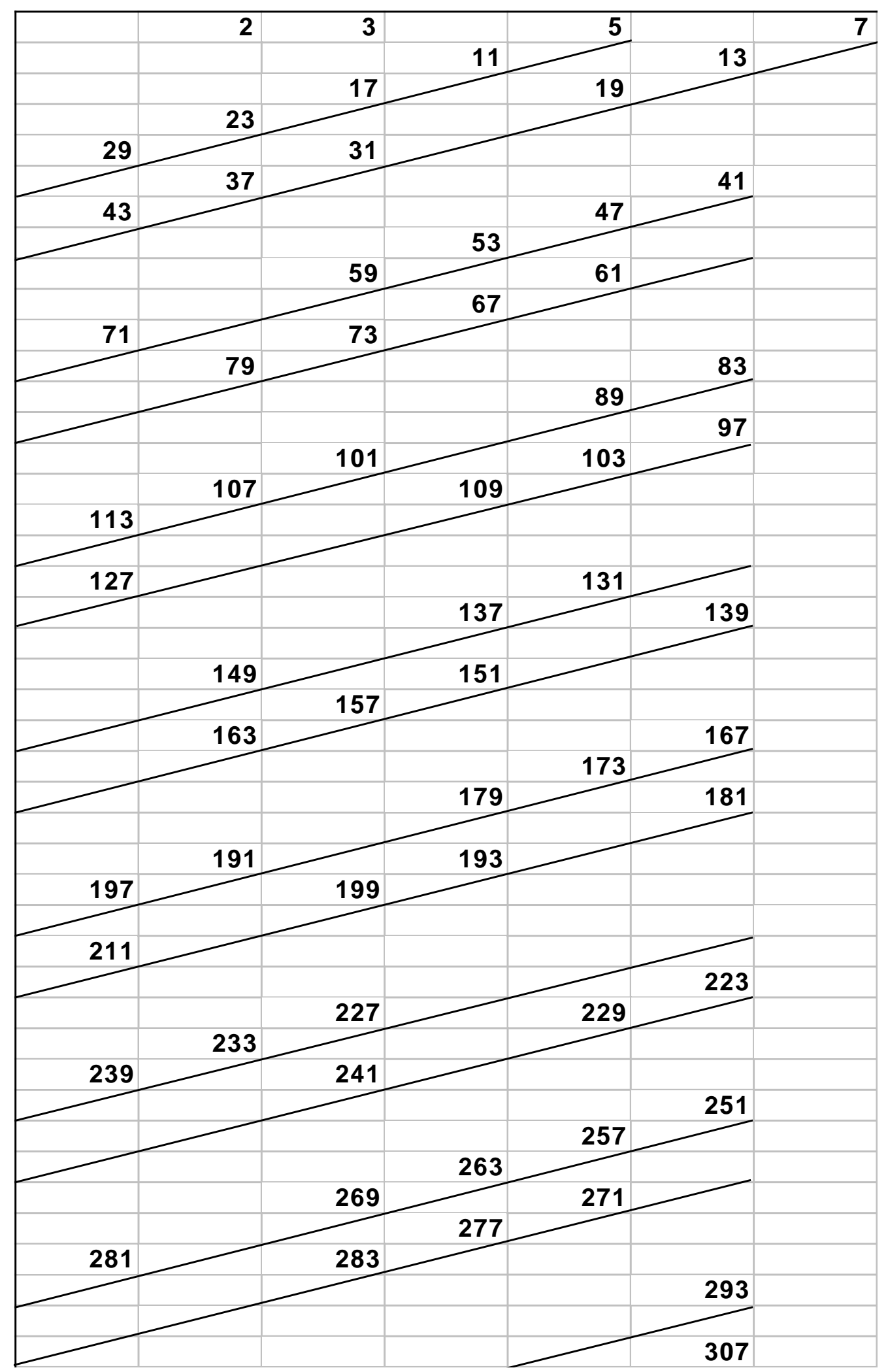

Figure 1. Seven column array in which prime numbers and prime products arrange themselves 
Table 1. Various values of s used to calculate $6 s-1$ and $6 s+1$

\begin{tabular}{|c|c|c|}
\hline $\mathrm{s}$ & $6 s-1$ & $6 s+1$ \\
\hline 1 & 5 & 7 \\
\hline 2 & 11 & 13 \\
\hline 3 & 17 & 19 \\
\hline 4 & 23 & $25=5 \times 5$ \\
\hline 5 & 29 & 31 \\
\hline 6 & $35=5 \times 7$ & 37 \\
\hline 7 & 41 & 43 \\
\hline 8 & 47 & $49=7 \times 7$ \\
\hline 9 & 53 & $55=5 \times 11$ \\
\hline 10 & 59 & 61 \\
\hline 11 & $65=5 \times 13$ & 67 \\
\hline 12 & 71 & 73 \\
\hline 13 & $77=7 \times 11$ & 79 \\
\hline 14 & 83 & $85=5 \times 17$ \\
\hline 15 & 89 & $91=7 \times 13$ \\
\hline 16 & $95=5 \times 19$ & 97 \\
\hline 17 & 101 & 103 \\
\hline 18 & 107 & 109 \\
\hline 19 & 113 & $115=5 \times 23$ \\
\hline 20 & $119=7 \times 17$ & $121=11 \times 11$ \\
\hline
\end{tabular}

\section{The Hyperbolic Representation}

The reason as to why this is given the attribute of 'hyperbolic' will be given later in this paper. Consider two integers D and $\mathrm{m}$, with $\mathrm{D}>\mathrm{m}$, and such that the smallest integer which will allow an equality consistent with $\mathrm{D}>\mathrm{m}$ is $\mathrm{D} \geq \mathrm{m}+1$. If $\mathrm{D}$ is an odd number, them $\mathrm{m}$ is even and if $\mathrm{D}$ is an even number, then $\mathrm{m}$ is an odd number. In addition, one or the other of $\mathrm{D}$ or $\mathrm{m}$ must be divisible by 3 , but not both. Now consider numbers $\mathrm{P}_{1}$ and $\mathrm{P}_{2}$ defined as:

$$
\begin{gathered}
P_{1}=D+m \\
P_{2}=D-m
\end{gathered}
$$


$P_{1} P_{2}=N$ which could be a prime number, a simple prime product, or a multiple prime product

$P_{1}$ can be a prime number, a simple prime product, or a multiple prime product.

$P_{2}$ can be a prime number, a simple prime product, a multiple prime product, or the identity element 1 , as a result of the above $D-m \geq 1$.(The Mathematician, 2010)

Examples are given in following Table 2:

Table 2. Various $\mathrm{D}$ and $\mathrm{m}$ values for various prime numbers and prime products

\begin{tabular}{|c|c|c|c|c|}
\hline $\mathrm{D}$ & $\mathrm{m}$ & $\mathrm{P}_{1}=\mathrm{D}+\mathrm{m}$ & $\mathrm{P}_{2}=\mathrm{D}-\mathrm{m}$ & $\mathrm{N}$ \\
\hline 3 & 2 & 5 & 1 & 5 \\
\hline 4 & 3 & 7 & 1 & 7 \\
\hline 13 & 12 & 25 & 1 & $25=5 \times 5$ \\
\hline 9 & 4 & 13 & 5 & 65 \\
\hline 30 & 11 & 41 & 19 & 779 \\
\hline 54 & 53 & 107 & 1 & 107 \\
\hline 12 & 1 & 13 & 11 & 143 \\
\hline 16 & 3 & 19 & 13 & 247 \\
\hline 181 & 180 & 361 & 1 & $361=19 \times 19$ \\
\hline 91 & 12 & 103 & 79 & 8137 \\
\hline 138 & 11 & 149 & 127 & 18923 \\
\hline 194 & 3 & 197 & 191 & 37627 \\
\hline 109 & 102 & 211 & 7 & 1477 \\
\hline 80 & 63 & $143=11 \times 13$ & 17 & 2431 \\
\hline 116 & 105 & $221=13 \times 17$ & 11 & 2431 \\
\hline 2816 & 2745 & $5561=67 \times 83$ & 71 & 394831 \\
\hline 442 & 225 & $667=29 \times 23$ & $217=31 \times 7$ & 144739 \\
\hline 45117 & 44834 & $89951=293 \times 307$ & 283 & 25456133 \\
\hline 41613 & 41306 & $82919=283 \times 293$ & 307 & 25456133 \\
\hline
\end{tabular}




\section{The Parabolic Representation}

The parabolic representation is related to the hyperbolic representation.

Consider the equation $\mathrm{y}=\mathrm{x}^{2}-2 \mathrm{Dx}+\mathrm{N}$, where $\mathrm{N}=(\mathrm{D}-\mathrm{m})(\mathrm{D}+\mathrm{m})$.

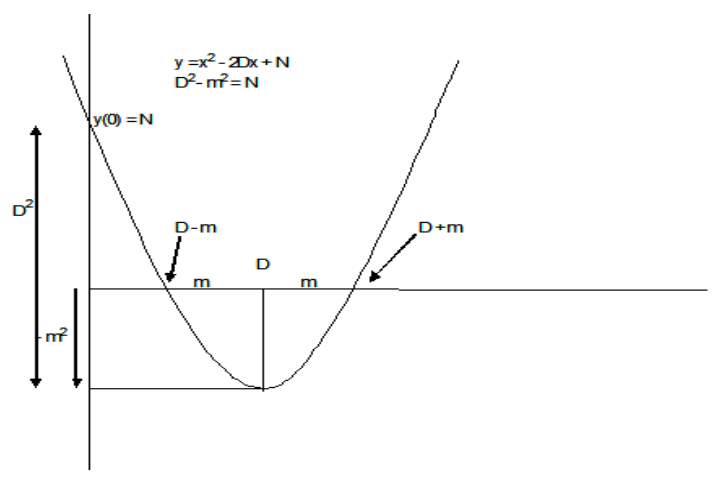

Figure 2. Parabolic representation

This is a parabola with solutions for $\mathrm{x}$ at $\mathrm{y}=0$.

\section{The Triangular Representation}

Fig. 3 illustrates the essentials below. The line of numbers starting with $25,36,49$, etc. is referred to by this author as the backbone, with yellow and turquoise ribs extending from it to numbers located in various cells. The numbers in these cells are the values of $\mathrm{N}$ (the prime product) for various prime numbers.

The triangular representation is related to the hyperbolic representation. Just as an introduction, (use the zoom function on your computer to view Fig. 3 or click Excel spreadsheet icon), find the number 253 which is the product of 11 and 23 . The number 253 sits on top of the dashed line $\mathrm{m}=6$. The dashed lines represent $\mathrm{H}_{2}$ or $6 \mathrm{~s}+1$, while the solid lines represent $\mathrm{H}_{1}$ or $6 s-1$. Notice how the dashed lines are spaced after every two solid lines, possibly indicating a regularity.

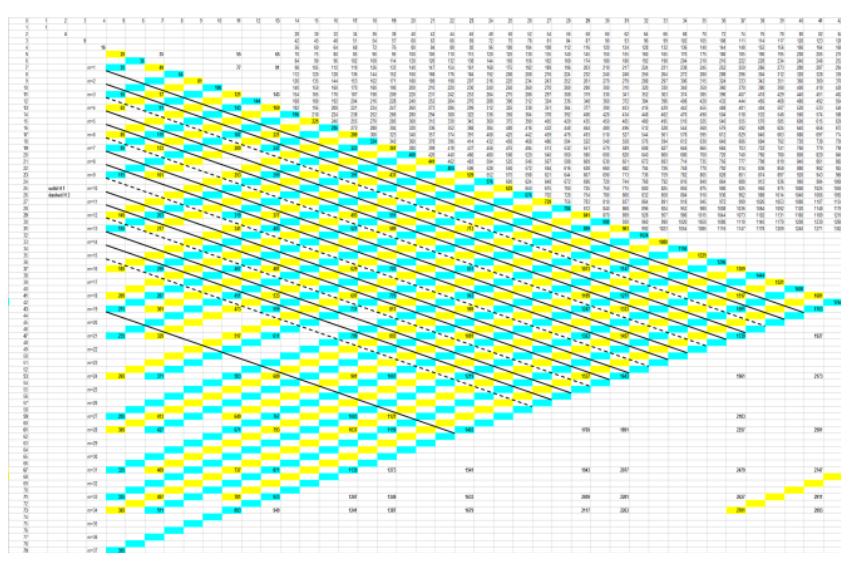

Figure 3. Exhibits relation of m's, $\mathrm{H}_{1}, \mathrm{H}_{2}$, prime numbers, and prime products

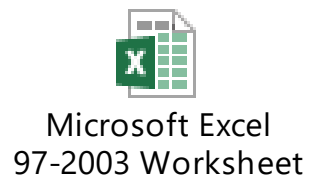




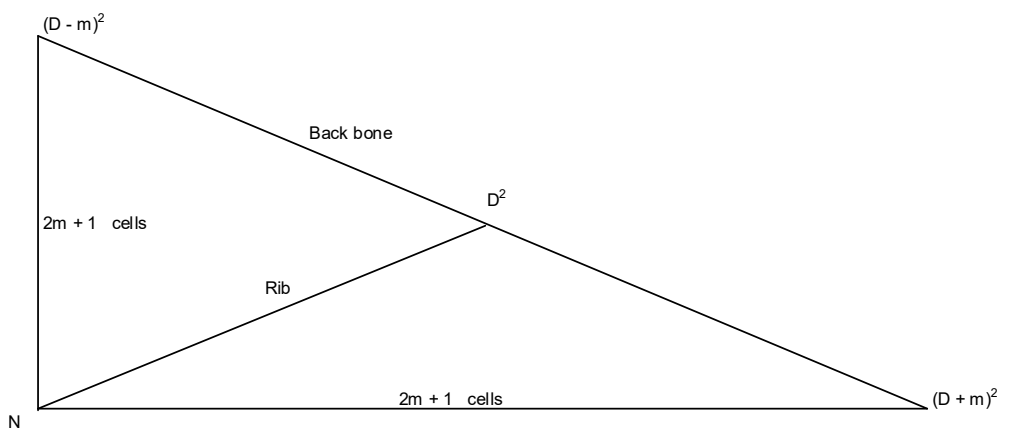

Figure 4. Generalized relationship between N, D - m, and D $+m$ in Figure 3

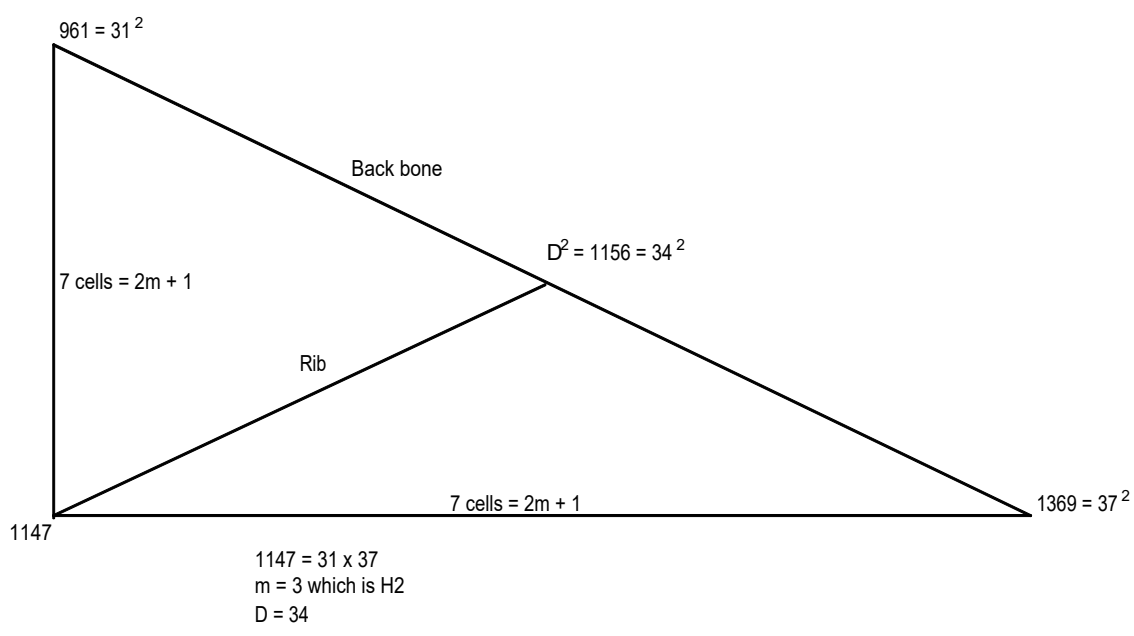

Figure 5. Specific example using $\mathrm{N}=1147$

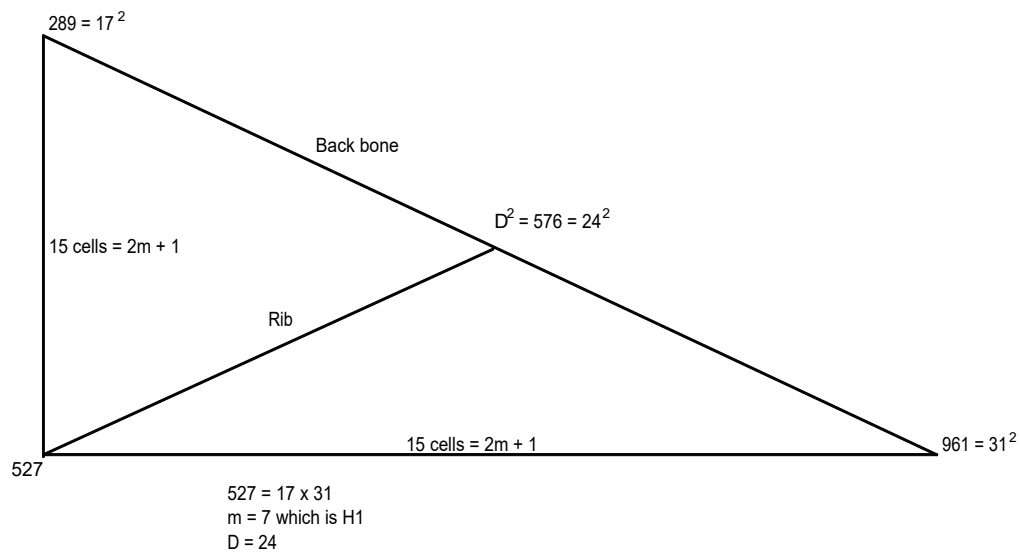

Figure 6. Specific example using $\mathrm{N}=527$ 


\section{Do These Two Primary Representations Have Anything in Common? (Bissonnet, P., 2017)}

One notices that the supposed prime numbers 2 and 3 are conspicuously absent from both representations. Let us consider first the hyperbolic representation in order to investigate the products of 2 and 3 with other prime numbers and prime products:

Table 3: Values of $\mathrm{D}$ and $\mathrm{m}$ using hyperbolic method for numbers 2 and 3

\begin{tabular}{|c|c|c|c|c|}
\hline $\mathrm{D}$ & $\mathrm{m}$ & $\mathrm{P}_{1}=\mathrm{D}+\mathrm{m}$ & $\mathrm{P}_{2}=\mathrm{D}-\mathrm{m}$ & $\mathrm{N}$ \\
\hline $3 / 2$ & $1 / 2$ & 2 & 1 & 2 \\
\hline 2 & 1 & 3 & 1 & 3 \\
\hline $7 / 2$ & $3 / 2$ & 5 & 2 & 10 \\
\hline 4 & 1 & 5 & 3 & 15 \\
\hline $9 / 2$ & $5 / 2$ & 7 & 2 & 14 \\
\hline 5 & 2 & 7 & 3 & 21 \\
\hline $13 / 2$ & $9 / 2$ & 11 & 2 & 22 \\
\hline 7 & 4 & 11 & 3 & 33 \\
\hline $15 / 2$ & $11 / 2$ & 13 & 2 & 26 \\
\hline 8 & 5 & 13 & 3 & 39 \\
\hline $19 / 2$ & $15 / 2$ & 17 & 2 & 34 \\
\hline 10 & 7 & 17 & 3 & 51 \\
\hline $21 / 2$ & $17 / 2$ & 19 & 2 & 38 \\
\hline 11 & 8 & 19 & 3 & 57 \\
\hline $25 / 2$ & $21 / 2$ & 23 & 2 & 46 \\
\hline 13 & 10 & 23 & 3 & 69 \\
\hline $27 / 2$ & $23 / 2$ & 25 & 2 & 50 \\
\hline 14 & 11 & 25 & 3 & 75 \\
\hline 19 & 16 & 35 & 3 & 105 \\
\hline
\end{tabular}

It is quite apparent from the above Table 3 that (1) the fractions violate the postulate that $\mathrm{D}$ and $\mathrm{m}$ are integers and (2) the entries which are indeed integer, one being odd and the other even, violate the postulate that one or the other must be divisible by 3 . Therefore, the hyperbolic representation does not accept that 2 and 3 are prime numbers.

Let us now consider the double helix representation. The comparison of 2 and 3 with the prime numbers beginning with 5 on Helix $1\left(\mathrm{H}_{1}\right)$ and 7 on Helix $2\left(\mathrm{H}_{2}\right)$ results in the following short tables of indicators that 2 and 3 are not true prime numbers: 
Characteristic 1: Obeys the classical definition of prime numbers?

\begin{tabular}{|l|l|l|l|l|l|l|l|l|l|l|l|}
\hline & 2 & 3 & 5 & 7 & 11 & 13 & 17 & 19 & 23 & 31 & Etc. \\
\hline Yes & $\sqrt{ }$ & $\sqrt{ }$ & $\sqrt{ }$ & $\sqrt{ }$ & $\sqrt{ }$ & $\sqrt{ }$ & $\sqrt{ }$ & $\sqrt{ }$ & $\sqrt{ }$ & $\sqrt{ }$ & \\
No & & & & & & & & & & & \\
\hline
\end{tabular}

Characteristic 2: Falls along either one or the other of $6 s-1$ or $6 s+1$, where $s \geq 1$ ?

\begin{tabular}{|l|l|l|l|l|l|l|l|l|l|l|l|}
\hline & 2 & 3 & 5 & 7 & 11 & 13 & 17 & 19 & 23 & 31 & Etc. \\
\hline Yes & & & $\sqrt{ }$ & $\sqrt{ }$ & $\sqrt{ }$ & $\sqrt{ }$ & $\sqrt{ }$ & $\sqrt{ }$ & $\sqrt{ }$ & $\sqrt{ }$ & \\
No & $\sqrt{ }$ & $\sqrt{ }$ & & & & & & & & & \\
\hline
\end{tabular}

Characteristic 3: Do products of prime numbers fall along $6 s-1$ or $6 s+1$, where $s \geq 1$ ?

\begin{tabular}{|c|c|c|c|c|c|c|c|c|c|c|c|c|}
\hline & $2 \times 2$ & $3 \times 3$ & $2 \times 3$ & $2 \times 5$ & $3 \times 5$ & $2 \times 11$ & $3 \times 11$ & $2 \times 7$ & $3 \times 7$ & $2 \times 13$ & $3 \times 13$ & Etc. \\
\hline Yes & & & & & & & & & & & & \\
No & $\sqrt{ }$ & $\sqrt{ }$ & $\sqrt{ }$ & $\sqrt{ }$ & $\sqrt{ }$ & $\sqrt{ }$ & $\sqrt{ }$ & $\sqrt{ }$ & $\sqrt{ }$ & $\sqrt{ }$ & $\sqrt{ }$ & \\
\hline
\end{tabular}

\begin{tabular}{|c|c|c|c|c|c|c|c|c|c|c|c|c|}
\hline & $5 \times 5$ & $7 \times 7$ & $5 \times 7$ & $5 \times 11$ & $7 \times 11$ & $5 \times 13$ & $7 \times 13$ & $11 \times 13$ & $5 \times 17$ & $7 \times 17$ & $17 \times 13$ & Etc. \\
\hline Yes & $\sqrt{ }$ & $\sqrt{ }$ & $\sqrt{ }$ & $\sqrt{ }$ & $\sqrt{ }$ & $\sqrt{ }$ & $\sqrt{ }$ & $\sqrt{ }$ & $\sqrt{ }$ & $\sqrt{ }$ & $\sqrt{ }$ & \\
& $\mathrm{H}_{2}$ & & & $\mathrm{H}_{1}$ & & $\mathrm{H}_{1}$ & & $\mathrm{H}_{1}$ & $\mathrm{H}_{2}$ & & $\mathrm{H}_{1}$ & \\
No & & & & & & & & & & & & \\
\hline
\end{tabular}

It is impossible for $6 s-1=2$ or $6 s+1=2$ or $6 s-1=3$ or $6 s+1=3$ and at the same time require that $s$ be an integer and satisfying $s \geq 1$. As shown in a previous paper (Bissonnet, P., 2017), $\mathrm{s}$ is a composite number which is equal to the sum of two other integers: $s=r+n$, upon referring to Fig. $1, r$ is the row number and $n$ is the complex of double lines, the first being $\mathrm{n}=0$, then $\mathrm{n}=1$, etc. $\mathrm{S}$ is definitely an integer with the minimum value of 1 .

Table 4. Shows that $\mathrm{s}$ is not an integer for prime numbers multiplied by 2 or 3

\begin{tabular}{|c|c|c|c|c|c|c|}
\hline $\begin{array}{c}\mathrm{N} \\
\text { prime number }\end{array}$ & $2 \mathrm{~N}$ & $\mathrm{~s}$ in $6 \mathrm{~s}-1$ & $\mathrm{~s}$ in $6 \mathrm{~s}+1$ & $3 \mathrm{~N}$ & $\mathrm{~s}$ in $6 \mathrm{~s}-1$ & $\mathrm{~s}$ in $6 \mathrm{~s}+1$ \\
\hline 53 & 106 & 17.833 & 17.500 & 159 & 26.667 & 26.333 \\
\hline 101 & 202 & 33.833 & 33.500 & 303 & 50.667 & 50.333 \\
\hline 109 & 218 & 36.500 & 36.167 & 327 & 54.667 & 54.333 \\
\hline 149 & 298 & 49.833 & 49.500 & 447 & 74.667 & 74.333 \\
\hline
\end{tabular}




\begin{tabular}{|c|c|c|c|c|c|c|}
\hline 157 & 314 & 52.500 & 52.167 & 471 & 78.667 & 78.333 \\
\hline 191 & 382 & 63.833 & 63.500 & 573 & 95.667 & 95.333 \\
\hline 211 & 422 & 70.500 & 70.167 & 633 & 105.667 & 105.333 \\
\hline 241 & 482 & 80.500 & 80.167 & 723 & 120.667 & 120.333 \\
\hline 283 & 566 & 94.500 & 94.167 & 849 & 141.667 & 141.333 \\
\hline 307 & 614 & 102.5 & 102.167 & 921 & 153.667 & 153.333 \\
\hline
\end{tabular}

Therefore, the double helix representation also rejects 2 and 3 as being true prime numbers.

\section{The Logic of 2 and 3 Not Being Prime Numbers}

If the double helix representation and the hyperbolic representation are true portals through which to observe prime numbers, simple prime products, and multiple prime products, then is it not a somewhat unsound and circular type of reasoning to assume that 2 and 3 are prime numbers, when such numbers show up in the very definitions/postulates of what is attempting to be proven or ascertained. Namely, the author is referring to the mention of 6 in $6 \mathrm{~s}-1$ and $6 \mathrm{~s}+1$. We know that 6 is 2 times 3 . What about the hyperbolic representation, where one of the requirements is that one or the other of $\mathrm{D}$ or $\mathrm{m}$ must be divisible by 3 ? The author believes that this is further mounting evidence for 2 and 3 not being true prime numbers.

\section{Conclusion (Bissonnet, P., 2011; Bissonnet, P., 2016)}

It is very disturbing that both of the primary representations (double helix and hyperbolic) seem to view prime numbers, as well as simple prime products and multiple prime products, on the same or equivalent mathematical footing. It is the dream of mathematicians to somehow separate or filter all prime numbers from the infinite variety of numbers. The current trend is to push further the goal of solving the Riemann Hypothesis. This author sees two very troubling difficulties to this goal; namely, as just stated (1) the seemingly equal mathematical footing given by nature to prime numbers and their products, and (2) the budding recognition (at least by this author) that 2 and 3 are not true prime numbers. In physics, there is a principle that tells an experimenter that there is a limit to how much information he can extract from a system, namely: the uncertainty principle. Whether or not there is a similar principle at work in mathematics is unknown, but it pays to be cautious just the same.

The hyperbolic representation was given the name of 'hyperbolic' for two reasons.

(1) Because of the following argument. Consider the form of

$$
D^{2}-m^{2}=N
$$

If we divide both sides by $\mathrm{N}$, we obtain

$$
\left(\frac{D}{\sqrt{N}}\right)^{2}-\left(\frac{m}{\sqrt{N}}\right)^{2}=1
$$

This is an important equation, because we recall from relativity theory the equation

$$
x^{2}-c^{2} t^{2}= \pm 1
$$

which represents the calibration hyperbola curves in special relativity. This means that, strangely, the values of $\left(\frac{D}{\sqrt{N}}\right)$

and $\left(\frac{m}{\sqrt{N}}\right)$ transfer to a new set of values $\left(\frac{D^{\prime}}{\sqrt{N^{\prime}}}\right)$ and $\left(\frac{m^{\prime}}{\sqrt{N^{\prime}}}\right)$

via a set of transformations similar to the Lorentz transformations, although the $\beta$ or velocity factor has little meaning. Could this imply that prime products might indicate a way that special relativity can be united with quantum mechanics, since quantum mechanics deals with integers in many instances, and this does seem to exemplify a way that integers 
might be connected with the Lorentz transformations.

(2) Let

$$
\begin{aligned}
& P(x)=D(x)+m(x) \\
& Q(x)=D(x)-m(x)
\end{aligned}
$$

where

$$
\begin{aligned}
& D(x)=\sqrt{N} \cosh x \\
& m(x)=\sqrt{N} \sinh x
\end{aligned}
$$

where $\cosh \mathrm{x}$ and $\sinh \mathrm{x}$ are hyperbolic cosine and sine respectively.

As a result of equation 4 , we arrive at a geometric identity

$$
\cosh ^{2}(x)-\sinh ^{2}(x)=1
$$

Consequently, we have the following conjecture:

\section{Conjecture:}

There is one and only one $x=x_{0}$ (not necessarily an integer) which causes both $D\left(x_{0}\right)$ and $m\left(x_{0}\right)$ to simultaneously assume integer status. In other words, $\sin D\left(x_{0}\right) \pi=0$ and $\sin m\left(x_{0}\right) \pi=0$.

Let us do some further supposing. To give a visual representation of equal mathematical footing, let us suppose that prime numbers can be represented by small green balls of a given diameter and weight. Suppose that simple prime products can also be represented by small green balls of the same color, diameter, and weight. The same for multiple prime products. How, then, can one form a sieve to sift out the prime numbers from the rest? Let us further suppose that the real physical world is built upon prime numbers, simple prime products, and multiple prime products. Consider that nature is as confused as we are in trying to separate prime numbers from the rest of the prime products and therefore exhibits an indeterminancy, delay, or whatever you want to call it in reaching a physical state. Could this not be considered an uncertainty? Could the uncertainty principle be considered the result of the mathematical equivalency of prime numbers, simple prime products, and multiple prime products in this type of physical world or physical reality; hence, the rudimentary genesis of quantum mechanical waves. Couple this with the relation of prime products with the Lorentz-like transformations, and we possibly have a way to unite special relativity and quantum mechanics. Quite a thought, isn't it?

From another point of view, let us reconsider a possible meaning of equal mathematical footing of prime numbers, simple prime products and multiple prime products in a physical reality built upon same. Consider the above green balls, which are indistinguishable, and which seemingly have no aversion to congregating together. Isn't this reminiscent of a class of particles called bosons? By the way, the author has no dislike for the numbers 2 or 3 . If 2 or 3 do not represent prime numbers, where could they fit in? Possibly 2 finds an exposition in two groups of particles called spin $1 / 2$ fermions and spin 2 gravitons. Possibly 3 finds an exposition in a triangular class of particles called quarks with charges of $+2 / 3,+2 / 3$ and $-1 / 3$ the charge of the electron.

\section{Acknowledgments}

My thanks to the anonymous reviewers' comments, for making this a better paper.

\section{References}

Bissonnet, P. (2006). Do Prime Numbers Obey a Three-Dimensional Double Helix? HadronicJournal, 29, 387-400.

Bissonnet, P. (2011). An Investigation into Reducing a Prime Product from Two Seemingly Independent Variables to Only One Independent Variable, Almanac of Modern Science and Education (A Russian Journal), 6(49), 57 - 58.

Bissonnet, P. (2016). Interesting Facts Concerning Prime Products and Their Relationship to Lorentz-Like Transformations, (unpublished). http://vixra.org/abs/1607.0381

Bissonnet, P. (2017). Addendum to Paper Entitled "Do Prime Numbers Obey a Three-Dimensional Double Helix?", Journal of Applied Mathematics and Physics, 5, 1825-1836. https://doi.org/10.4236/jamp.2017.59154

Hahn, H. K. (2008). About the Logic of the Prime Number Distribution, retrieved from https://arxiv.org/abs/0801.4049 .

The Mathematician (2010). Why is the number 1 not considered a prime number? Ask a Mathematician/Ask a Physicist (on-line question and answer). www.askamathematician.com. 


\section{Copyrights}

Copyright for this article is retained by the author(s), with first publication rights granted to the journal.

This is an open-access article distributed under the terms and conditions of the Creative Commons Attribution license (http://creativecommons.org/licenses/by/4.0/). 